\title{
Hydrochlorothiazide Induced Vasculitis
}

\section{Rachael Hauser M.D.; Elisha Brumfield D.O.}

\section{Introduction}

- Cutaneous leukocytoclastic angiitis, or hypersensitivity vasculitis, can result from treatment by many commonly prescribed medications

- Patients present with various manifestations which may delay diagnosis and appropriate treatment

\section{Case Presentation}

- History: 34 year-old previously healthy Hispanic female was transferred from an outlying hospital for concerns of progressive rash of two weeks duration with lower extremity edema, severe pruritus, arthralgia, abdominal pain

- Past medical history: newly diagnosed with hypertension, treated with Hydrochlorothiazide for one month

- Laboratory: UA, CBC, ANCA, ESR, Complement, RAF, ANA Panel, Cryoglobulin, HIV, Hepatitis studies were normal

- Cutaneous Biopsy: Inflammatory infiltration of lymphocytes and neutrophils, extravasation of erythrocytes, fibrin deposition in the walls of the small blood vessels with no evidence of IgA antibodies by direct immunofluorescence

- Treatment: discontinued Hydrochlorothiazide, began high dose IV steroid therapy with transition to oral taper

- Follow up: patient revealed prolonged recovery with continued hyperpigmented lesions and peripheral neuropathy

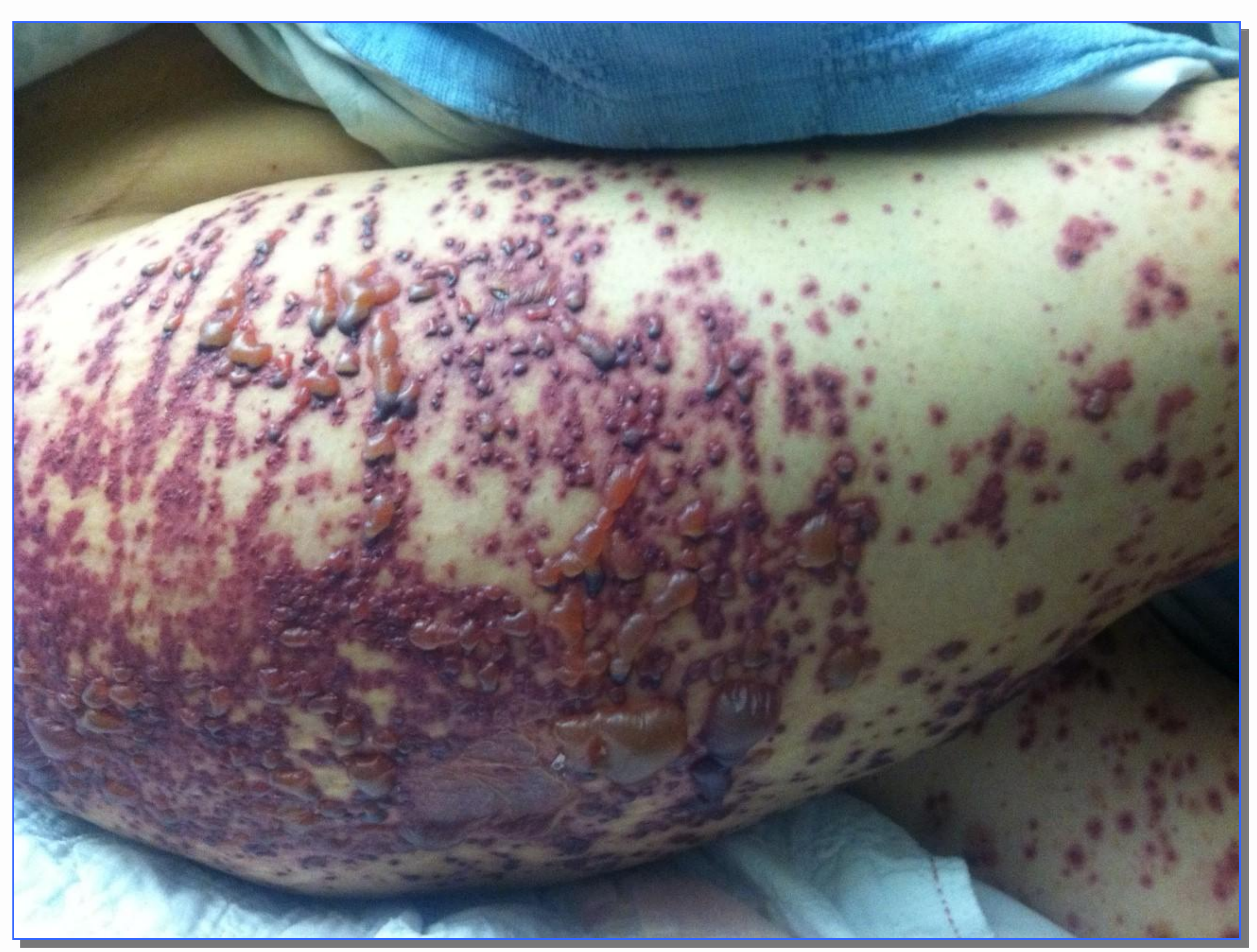

Palpable Purpura with Hemorrhagic Bullae

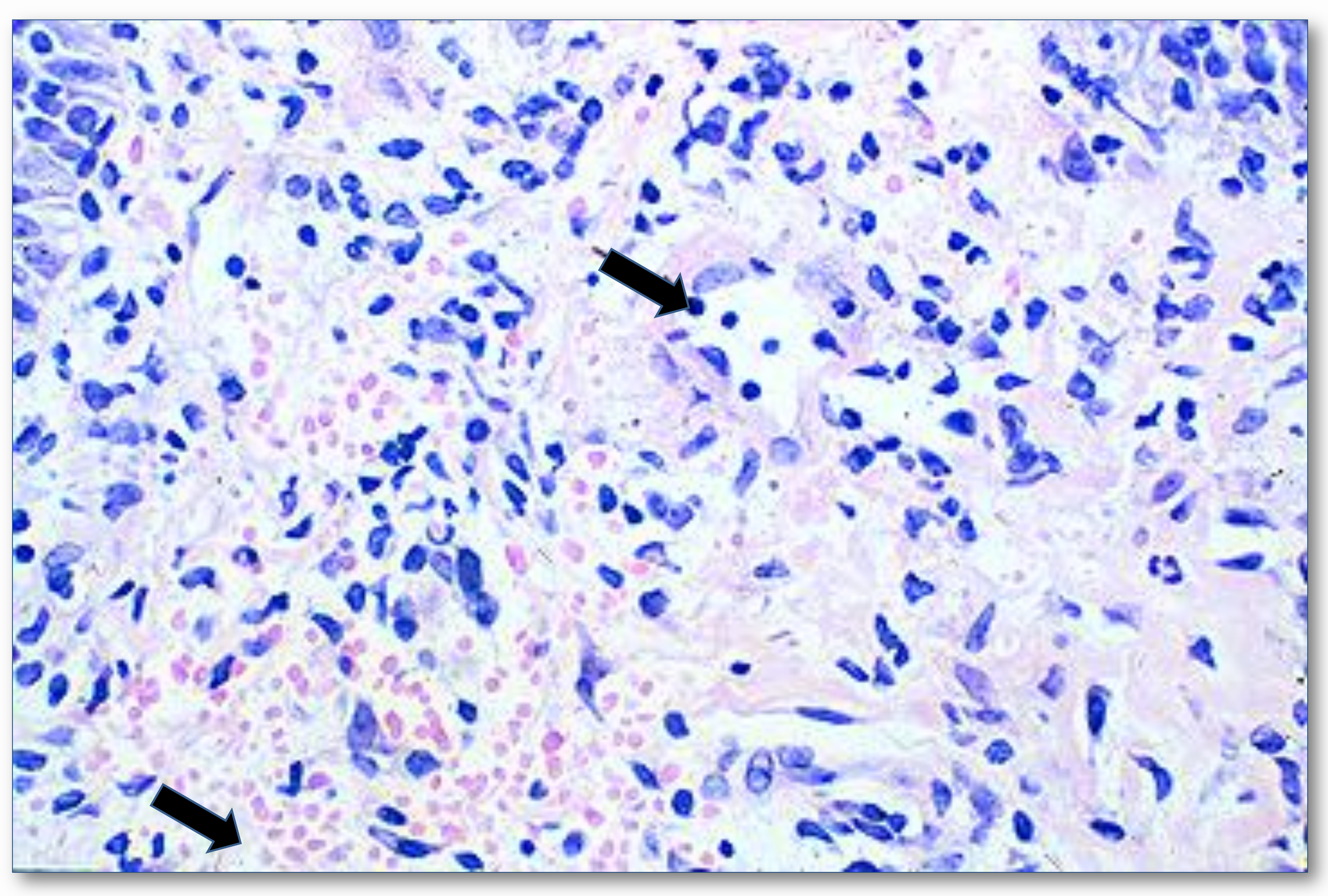

Punch Biopsy:

Extravasation of lymphocytes and erythrocytes.

\section{References}

1. Tosca N, Stratigos JD. Possible pathogenetic mechanisms in allergic cutaneous vasculitis. Int J Dermatol 1988;27(5):291-6.

2. Calabrese LH, Michel BA, Bloch DA, et al. The American College of Rheumatology 1990 criteria for the classification of hypersensitivity vasculitis. Arthritis Rheum 1990;33(8):1108-13.

3. Calabrese LH, Duna GF. Drug-induced vasculitis. Curr Opin Rheumatol 1996;8(1):34-40.

\section{Discussion}

- Hypersensitivity vasculitis is a clinical syndrome due to immune complex deposition in capillaries, venules, and arterioles ${ }^{1}$

- Clinical symptoms are most commonly palpable purpura, but also includes arthralgia, myalgia, fever

- Latent period typically 7-10 days after exposure to offending agent

- Diagnostic Criteria ${ }^{2}$

$$
\text { Age }>16
$$

Offending agent

Palpable purpura

Maculopapular rash

Biopsy with neutrophils around arterioles/venules

- Common insulting medications include penicillin, cephalosporins, loop diuretics, and thiazide diuretics

- These agents function as a hapten to stimulate an immune response ${ }^{3}$

- Infections such as hepatitis and HIV have also been associated

- Although an infrequent adverse reaction to a regularly prescribed medication, providers must be aware of this risk to provide appropriate treatment and prevent long term sequelae

\section{KU}

SCHOOL OF MEDICINE MNOPTHA

The University of Kansas 